Agrostis or Agrostis-Festuca, liable to invasion by bracken, gorse, etc., and much in need of liming, manuring, draining or re-seeding. $8,281,000$ acres or $22 \cdot 1$ per cent, in addition to which there are $2,010,000$ acres or $5 \cdot 3$ per cent with an admixture of better land.

$G$ 7. Poor Quality Heavy Land. Land with very heavy, intractable soils or low-lying and difficult to drain. Poor grassland, especially Agrostis infested by rushes (Juncus). 382,500 acres or $1 \cdot 0$ per cent, together with 988,000 acres $(2 \cdot 6$ per cent) of which parts have already been improved. This does not, of course, include the huge areas of $A G 6$ and $G 4$ land badly in need of better drainage-probably half of the whole. Medium quality farm land (categories 5,6 and 7) thus totals $14,257,000$ acres or 38.0 per cent of England and Wales.

$H$ 8. Poor Quality Mountain Land (Mountain or Rough Hill Pastures). Natural and semi-natural vegetation covering much of the uplands of old rocks especially above 1,000 feet. Thin, poor, stony, immature soils, usually leached and podsolic ; in areas of impeded drainage, acid peats ; in some areas many rock outcrops. Vegetation permits a detailed classification (leading types are Nardus-Molinia moors, Eriophorum moors, heather moors, bilberry moors, etc.) and gives a clue to possibilities of improvement and use. 3,735,000 acres or $9 \cdot 9$ per cent, together with an additional 723,500 acres or 1.9 per cent with improved patches.

H 9. Poor Quality Light Land (Lowland Heaths and Moors). Very light scundy or gravelly podsolized soils usually too 'hungry' (that is, unretentive of manures) for economic cultivation. Usually heath- land ; suitable for coniferous afforestation or use as open spaces. Includes also some undrained fens and mosses. 316,000 acres or 0.8 per cent, in addition to $1,200,000$ or $3 \cdot 2$ per cent mixed with better land.

$H$ 10. Poorest Land (Saltings, Rough Marsh Pas. ture, Shingle, etc.). Land of varied character, but in its present state of little agricultural value, though some (salt marshes) could be reclaimed. 78,000 acres or 0.2 per cent.

As matters of national policy it is suggested: (a) that the first four categories, that is, the best agricultural lands, be preserved so far as possible in agricultural use; $(b)$ that major schemes using large areas of land-for green belts, national parks, afforestation, etc.- - be restricted to the poorer types of land. Other developments such as new trunk roads of the parkway type can well be aligned accordingly, while some of the poorest land (for example, salt marshes) is well situated for heavy industry ; $(c)$ that the integration of land use envisaged by town planning schemes, the establishment of garden cities, etc., is especially important on land of intermediate quality.

The above figures are provisional estimates and include the Isle of Man. Closely built-over areas such as the centres of cities and industrial works are excluded but land already occupied by houses with gardens is included. Calculations of the areas of the latter are being made and the map referred to is now in the Press.

\footnotetext{
${ }^{1}$ See, for example, Town and Country Planning, March 1941, where this principle is stressed by the writers of four articles out of five published in that issue.

. Summarized in NATURE, 143, 456 (1939).
}

\title{
STRUCTURE OF MOLECULES AND AGGREGATES OF MOLECULES
}

\begin{abstract}
A SYMPOSIUM on the structure of molecules and aggregates of molecules was held at Columbia University during December 30-January I, 194I, under the auspices of the Division of Physical and Inorganic Chemistry of the American Chemical Society. Some twenty papers which were submitted at this meeting appear in extenso in the January numbers of the Journal of Chemical Physics and the Journal of the American Chemical Society.

In a short introductory paper, Prof. J. G. Kirkwood, chairman of the organizing committee, points out that "modern physical chemistry is primarily concerned with problems of structure as related both to the properties of systems in equilibrium and to the mechanism of the physical and chemical processes traversed in the attainment of equilibrium". A large variety of theoretical and experimental techniques are, therefore, required to enable the determination of the structure of molecules of vastly different complexities, the structure of molecular aggregates, the nature and magnitude of intermolecular forees, the spatial arrangement of atoms in a molecule or of molecules in an aggregate, interatomic distances, and many other properties. On the theoretical side quantum mechanics lead to a fuller understanding of individual molecules, and aggregates of molecules are being investigated to a very great extent by the methods of statistical mechanics. On the experimental side the structure of simple molecules can be
\end{abstract}

elucidated by a variety of well-established methods ; but to investigate the structure of solid and liquid aggregates is more difficult and, of the available methods, X-ray methods appear to give the fullest information. Thermodynamic properties also shed light on structural theories of gases, but with liquids and solids the evidence from this source is less complete and less conclusive; nevertheless, increasingly promising results on these latter types are accruing from their statistical mechanical treatment.

The papers, presented at the symposium and briefly summarized here, illustrate the width and variety of the topic and the modern means of tackling its immense unsolved mysteries.

Among the purely theoretical contributions is one by Mayer and Montroll, who have put forward definitions of functions capable of describing the distribution of molecules in a system and have derived equations for their evaluation. When applied to gases, the distribution functions can be developed in power series in fugacity or inverse volume in which the coeffieients are integrals involving the mutual potential energy between pairs of molecules. Extension to condensed systems, although possible, renders the equations much more complicated, but their approximate solution can be achieved by application of the cell method involving the probabilities of population of virtual cells.

Quite a different application of statistical mechanics 
is made by J. C. Slater in attempting to explain the transition in $\mathrm{KH}_{2} \mathrm{PO}_{4}$. The crystal structure indicates that the $\mathrm{PO}_{4}$ groups are connected by hydrogen bonds, and thus different orientations of the $\mathrm{H}_{2} \mathrm{PO}_{4}{ }^{\prime}$ dipoles will arise from different possible arrangements of the hydrogens. When the $\mathrm{H}_{2} \mathrm{PO}_{4}^{\prime}$ groups are pointing along the axis of the crystal they will have lowest energy, and spontaneous polarization along his axis will occur causing polarization below the Curie point. The statistical mechanical treatment predicts for this transition a phase change of the first order with a sudden change from the lowtemperature polarized state to the high-temperature unpolarized state. Experimentally it has been found that the transition is of the second order and is confined to a much narrower temperature range than that predicted by the Weiss theory. But this may be due to broadening of a first-order phase change rising from irregular shifts of transition temperatures in certain regions of the crystal produced by stresses and deformation at temperatures below the transition point. It has been found, too, that the susceptibility above the Curie point, calculated by the statistical mechanical method, agrees, unlike the predicted value on the Weiss theory, with the experimental result. On the other hand, the calculated entropy change is unaccountably 0.11 units less than the observed value.

The existing qualitative ideas regarding hyperconjugation, that is, the conjugation of a group such as $\mathrm{CH}_{3}$ with groups containing double or triple bonds, are put on a quantitative basis by a quantummechanical treatment by $\mathrm{R}$. S. Mulliken et al.* Hyperconjugation is considered to be omnipresent. Three general types of conjugation are recognized: (a) first order conjugation, for example, $\mathrm{HC} \equiv \mathrm{C}-\mathrm{C} \equiv$ $\mathrm{CH}$; (b) second order conjugation (first order hyperconjugation), for example, $\mathrm{H}_{3} \equiv \mathrm{C}-\mathrm{C} \equiv \mathrm{H}$; (c) third order conjugation (second order hyperconjugation), for example, $\mathrm{H}_{3} \equiv \mathrm{C}-\mathrm{C} \equiv \mathrm{H}_{3}$. Hyperconjugation stabilizes molecules. Bonds are of two types, donor or acceptor; $\mathrm{C}-\mathrm{H}$ bonds in saturated hydrocarbons are donor and $\mathrm{C}-\mathrm{C}$ bonds acceptor. As a result of type (c) conjugation acceptor bonds gain appreciably in stability whereas donor bonds lose only a small amount of stability. Hyperconjugation confers on $\mathrm{C}-\mathrm{C}$ bonds approximately $1 \mathrm{I}$ per cent double bond character and on $\mathrm{C}=\mathrm{C}$ bonds about 12 per cent triple bond character. The effects of conjugation in various molecules receive detailed discussion.

Debye, in 1925, explained the salting out of nonelectrolytes by their electrostatic interaction with ions, and treated the non-electrolyte as a homogeneous medium. This method is extended and applied to the whole range of composition of alcoholwater mixtures by Scatchard, who takes account of the heterogeneity of the solvent. Satisfactory agreement with experimental data was not obtained probably because account was not taken of the actual structure of the solvent.

The ascertainment of structure is possible by a variety of methods, and the symposium contains several papers on experimental and theoretical aspects of this topic. Beach, in continuation of his electron diffraction studies, contributes a paper dealing with the structure and dimensions of dioxadiene, $p$-dioxene, and furan and two of its derivatives. In electron diffraction work information about the intensity distribution of scattered electrons is at

- Papers published in Journal of the American Chemical Society. present not very readily obtained. Recent improvements in technique, for example, the introduction of the rotating sector, should soon yield experimental information on this point. It should then be possible not only to determine interatomic distances but also to ascertain their natural degree of indeterminacy. The problem is treated theoretically by Debye by considering the influences of atomic vibrations and of free and hindered rotation of molecular groups on the scattering properties.

The application of X-ray spectroseopy is made by Hoard and Martin* to the structure of oxyfluoniobates. Interaction between aqueous solutions of niobium pentafluoride, $\mathrm{NbF}_{5}$, and potassium fluoride, $\mathrm{KF}$, gives either $\mathrm{K}_{2} \mathrm{NbOF}_{5} \mathrm{H}_{2} \mathrm{O}$ or $\mathrm{K}_{3} \mathrm{NbOF}_{6}$; and, if $\mathrm{HF}$ is added as a third reactant, $\mathrm{K}_{3} \mathrm{HNbOF}_{7}$ or $\mathrm{K}_{2} \mathrm{NbF}_{7}$. These are all crystalline compounds and their preparation, crystal forms, and the structures of the different ions are discussed, particularly $\mathrm{K}_{3} \mathrm{HNbO}_{7}$ which is found to be an aggregate of $\mathrm{K} \cdot \mathrm{HF}^{\prime}$, and octahedral $\mathrm{NbOF}_{5}$ " ions, having the salt-like structure $\mathrm{K}_{2} \mathrm{NbOF}_{5} . \mathrm{KHF}_{2}$ and isomorphous with $\mathrm{K}_{2} \mathrm{SnF}_{6} . \mathrm{KHF}_{2}$ and $\mathrm{K}_{2} \mathrm{PbF}_{6} \cdot \mathrm{KHF}_{2}$. On the theoretical side Huggins extends Bragg's photographic method for making a Fourier summation from crystal diffraction data.

Analysis of vibrational spectra of polyatomic molecules is difficult but there are several aids available. An important one is the evaluation of force constants by means of normal co-ordinate calculations. Using a valency-force potential these force constants may be employed to study the character of different bonds and their variation with environment. Crawford and Brinkley examine this problem with reference to the six related molecules $\mathrm{HCN}, \mathrm{C}_{2} \mathrm{H}_{2}, \mathrm{C}_{2} \mathrm{H}_{6}, \mathrm{CH}_{3} \mathrm{CN}$, methyl- and dimethyl-acetylene, and they show that stretching constants vary with bond type and with bond length, whereas bending and interaction constants vary only with bond type. To reduce the work of calculating vibration frequencies of complex molecules, Wilson develops various schemes and applies them to the methyl chloride molecule. $\mathrm{He}$ derives from these developments a modification of the Teller-Redlich isotopic product theorem. Morse functions, usually calculated from the energy of dissociation, nuclear separation, and fundamental vibration frequency, have been calculated by Hulbert and Hirschfelder, taking into account the additional spectroscopic constants, $\omega_{e} x_{e}$ and $\alpha_{e}$, and the method is applied to twenty-five diatomic molecules belonging to various classes, for example, hydrogen molecules, hydrides, halogens, oxides, alkalis. The new fiveparameter curves give potentials which lie above the Morse curves and indicate that the corrections for moderately large internuclear distances may amount to 10 per cent of the energy of dissociation. Where comparison is possible there is good agreement between observed and calculated potentials.

Smyth* discusses induction, resonance, and dipole moments and assesses contributions to dipole moments from inductive effects and resonance structures for a variety of molecules. He also computes the amount of ionic character in various bonds and obtains values in good agreement with electronegativities derived from thermal data. Comparison of the amounts of double bond character in carbon-halogen bonds in monohalogenobenzenes calculated from dipole moment data with those calculated from interatomic distances reveals wide differences and leads to the conclusion that the amounts of resonance contribu. tions to dipole moment values cannot be accurately ascertained. The significance of this conclusion may 
be somewhat altered by the revised values of the covalent radii of $\mathrm{H}, \mathrm{O}, \mathrm{N}$, and $\mathrm{F}$ given by Schomaker and Stevenson* who also show that the bond length between two atoms $A$ and $B, r_{A B}$ is less than the sum of the normal single covalent bond radii, $r_{A}+r_{B}$, by 0.09 times the difference between the electronegativities of $A$ and $B$.

Groups surrounding a chromophoric group exercise profound influences on the group and may even induce electronic transitions resulting in optical activity. These "vicinal actions" are discussed by Eyring and" Kauzmann by reference to a large variety of substances, and they show that optical rotatory power may be utilized in investigating the structure of molecules, provided that due account is taken of "vicinal actions" in calculating optical rotations.

Van Vleck obtains expressions for the susceptibility of an antiferromagnetic medium by means of the Debye vector model and finds tolerable agreement with experimental data. O. K. Rice discusses the equation of state for argon and derives its potential energy curve. The uncertainty as to whether it is the total potential of the electrode or only the overvoltage that is involved in the discharge of gaseous ions has been resolved by Kimball et al., who postulate the existence of two different electrical double layers at the electrode surface and two corresponding energy barriers over which protons must pass. What these barriers correspond with cannot be unequivoc. ally established, but it appears that most probably both correspond to proton transfers from water molecule to water molecule.

Rabinowitch and Epstein* discuss the polymerization of dyestuffs in solution from absorption spectra measurements on thionene and methylene blue. Organic dyestuffs show a universal property of dimerization quantitatively interpretable by London's theory of intermolecular forces. The physical chem. istry of insulin is investigated by Cohn et al. using the radioactive isotope of zinc $(\mathrm{Zn} *)$ and studying the influences of excess $\mathrm{Zn}^{*}, p \mathrm{H}$ and ionic strength on the crystallization and solubility of insulin.

The simultaneous appearance of these papers will prove of immense value to all interested in modern physical chemistry. The topic has so many varied aspects that it calls for large numbers of investigators, each a specialist in his own field. Co-ordination of the contributions of the various specialists is necessary to enable the 'ordinary' physical chemist to appreciate to the full the significance of the various contributions. It therefore seems a pity that the whole symposium was not published in one journal and that the discussions on the papers were not published along with them.

\section{CARNEGIE INSTITUTION OF WASHINGTON}

$$
\mathrm{Y}^{\mathrm{H}}
$$

EAR BOOK No. 39 of the Carnegie Institution at

Washingten covers the year July 1, 1939-June 30,1940 . In addition to the report of the President, it includes reports on the departmental activities and co-operative studies covering astronomy, terrestrial sciences, biological sciences and historical research.

The President, in his report, refers to the intensification of scientific activities in the United States even in fields which are remote from the National Defence Programme. This he attributes to the stimulation of a democracy by the existence of danger, and suggests that fundamental scientific advance, like basic intellectual accomplishment of many kinds, is often accelerated rather than retarded by national stress. The income from endowment continued to decrease, but the eurrent situation was transformed during the year by an emergency grant of 750,000 dollars from the Carnegie Corporation of New York, payable at the rate of 150,000 dollars annually for five years, and the year closed with a substantial excess of income over estimate.

The Geophysical Laboratory and the Depart. ment of Terrestrial Magnetism have co-operated during the year in a programme which substantially extends the limits of experimental work on the properties of materials under enormous pressures, utilizing a cascaded pressure equipment with which pressures of 200,000 atmospheres have already been reached, and certain physical measurements can be conducted with material under this pressure. Hightemperature studies of anhydrous systems and their presentation by equilibrium diagrams have been continued in the Geophysical Laboratory, as well as studies of equilibrium in systems containing water at various pressures and temperatures. The Department of Terrestrial Magnetism has also continued its studies on the ionosphere as well as its geomagnetic studies on fluctuations in solar radiation, while its activities in nuclear physics have been extended to include a programme of fundamental work in biology and chemistry utilizing the artificial radioactive isotopes of ordinary elements as tracers for following various reactions. This programme centres round a large cyclotron installed last year. Observations at the Mount Wilson Observatory have afforded definite evidence of the presence of organic compounds in interstellar space, and the evidence for the hydrocarbon gas $\mathrm{CH}$ and for cyanogen $\mathrm{CN}$ is almost conclusive. The Department of Genetics has investigated the mechanism by which cosmic rays as well as $\mathrm{X}$-rays cause mutations and hence the appearance of individuals differing abruptly from their ancestors. It has been found that very considerable delay may occur between the fracture of a chromosome and its rearrangement so that reproduction may proceed. Experimental modification of the process, for example, by the use of alkaloids to cause the doubling of chromosomes, yielded much new knowledge. The report includes a review of this work, as well as an account of endocrine studies in the same Department. Biochemical investigations in the Division of Plant Biology have covered the organic nutrition of plants, the use of radioactive carbon dioxide in photosynthesis, and oxidation, reduction and reactions in killed leaves. The report also includes an account of work by Robert Emerson and C. M. Lewis on the quantum efficieney of photosynthesis and the absorption of carbon dioxide in the light and in the dark. Work by the Department of Embryology has covered the physiology of the reproductive system, the endocrine organs, tissue culture and tumour studies, as well as investigations on the nervous system. The report also includes a brief account of investigations in the Nutrition Laboratory and by the Division of Historical Research. 